\title{
Towards Chemical Kinetics for Operando Electron Microscopy of Catalysts: 3D Modeling of Product Gas Distributions and Temperature Profiles During Catalysis
}

\author{
Joshua Vincent ${ }^{1}$, Jarod Vance ${ }^{1}$, Jayse Langdon ${ }^{1}$, Benjamin Miller ${ }^{2}$ and Peter Crozier ${ }^{1}$ \\ ${ }^{1}$ Arizona State University, Tempe, Arizona, United States, ${ }^{2}$ Gatan, Inc., Gilbert, Arizona, United States
}

In situ ETEM is a powerful tool for studying catalysts in the presence of gaseous reactants and applied heat. Modern instruments offer spatial resolutions better than $0.1 \mathrm{~nm}$, and the ability to visualize atomicscale dynamics under reaction conditions has led to insight into a wide breadth of catalytic processes [1]. Recently, modified specimen preparation methods have enabled measurable catalytic conversions to be generated in an open-cell ETEM, allowing for operando correlations of catalyst structure with activity $[2,3]$. In this approach, catalyst particles are dispersed on a porous glass fiber pellet and a metal grid. Both the pellet and grid are loaded into a furnace-style heating holder. As the catalyst-loaded furnace reactor is heated in the presence of gaseous reactants, catalysis occurs. The composition of product gases can be quantified with electron energy-loss spectroscopy (EELS). This operando pellet reactor approach offers an exciting opportunity to leverage quantitative chemical kinetic analysis during atomic-resolution TEM imaging. However, to reliably evaluate kinetics, it is necessary to establish a model of the reactor and assess the influence of any chemical or thermal gradients. Here, we develop a finite element model of the operando pellet reactor and determine the gas and temperature profiles during an operando TEM experiment to enable quantitative determination of chemical kinetics.

The COMSOL® Multiphysics software was used to model a FEI Titan ETEM and Gatan 628 single-tilt Ta heater holder. The model is applied to a $\mathrm{SiO}_{2}$-supported $\mathrm{Ru}$ catalyst performing $\mathrm{CO}$ oxidation. The Computational Fluid Dynamics, Heat Transfer, and Chemical Reaction Engineering modules were used. Bulk fluid flow is modeled with the Navier-Stokes equation, while multi-component diffusion is modeled with the Maxwell-Stefan equation. Heat transfer considerations include conduction, convection, and radiation. The catalytic reaction was modeled as irreversible and elementary, with an activation energy of $90 \mathrm{~kJ} / \mathrm{mol}$. The catalyst in the pellet was modeled with an egg-shell distribution. The Arrhenius preexponential factor, $A$, was adjusted until a match was achieved for experimentally measured conversions at $340{ }^{\circ} \mathrm{C}$ (here, $A=7 * 10^{12} \mathrm{~s}^{-1}$ ). Material properties and thermo-physical parameters were taken from tabulated sources. Steady-state simulations were performed for an inflow of 1 SCCM of stoichiometric $\mathrm{CO}$ and $\mathrm{O}_{2}$, which leads to a cell pressure of 3 mbar; the furnace was set in the range of $25-340{ }^{\circ} \mathrm{C}$.

The model geometry is shown in Figure 1, which displays the environmental cell (a) in full view and (b) in an enhanced view of the operando pellet reactor. Gas flows into the cell from the inlet on the left. The apertures in the pole pieces serve as outlets. A thermocouple on the furnace controls the reactor temperature. The gas composition measured by EELS is simulated with a line integral along the path labeled "EELS Line" in Figure 1b. The composition at the grid is found through a surface integral on the plane labeled "TEM Grid," and the composition in the pellet is calculated as an integral over its volume.

Figure 2a displays the 3D distribution of catalytically-produced $\mathrm{CO}_{2}$ in and around the reactor for a furnace set point of $230^{\circ} \mathrm{C}$. The simulated $\mathrm{CO}$ conversion $\left(\mathrm{X}_{C O}\right)$ at this condition is $34 \%$. The $\mathrm{CO}_{2}$ appears well-distributed in the gas phase throughout the cell, with a slight enhancement in the operando pellet at the top region enclosed by the impermeable furnace and Ta washer. The simulated 3D temperature profile (not shown here) is largely uniform throughout the entire furnace reactor, which agrees with prior furnaceholder modeling studies done by Mortensen et al. [4]. Figure 2b shows a quantitative comparison of the 
$\mathrm{CO}_{2}$ mole fraction at different domains across a range of conversion. Composition measurements simulated here agree well with experimentally acquired data (red boxes). Compositional inhomogeneities are observed, in particular between the composition in the pellet and that measured with EELS; however, it is noted that the difference between the EELS measurement and the composition at the TEM grid is smaller $\left(\sim 0.01-0.04\right.$ for $X_{C O}$ below 60\%). The fact that there is little discrepancy between the composition measured with EELS and that at the imaged catalyst is an important result which demonstrates that, under low conversion conditions, one may use EELS reliably to measure the apparent reaction rate at the TEM grid. Furthermore, the well-mixed composition in the gas phase suggests that the operando pellet architecture may approximate a continuously-stirred tank reactor (CSTR) [5]. The ability to apply a simple reactor model to the operando TEM data would greatly facilitate the evaluation of kinetic parameters (e.g., activation energies) for catalytic structure-activity relationships [6].
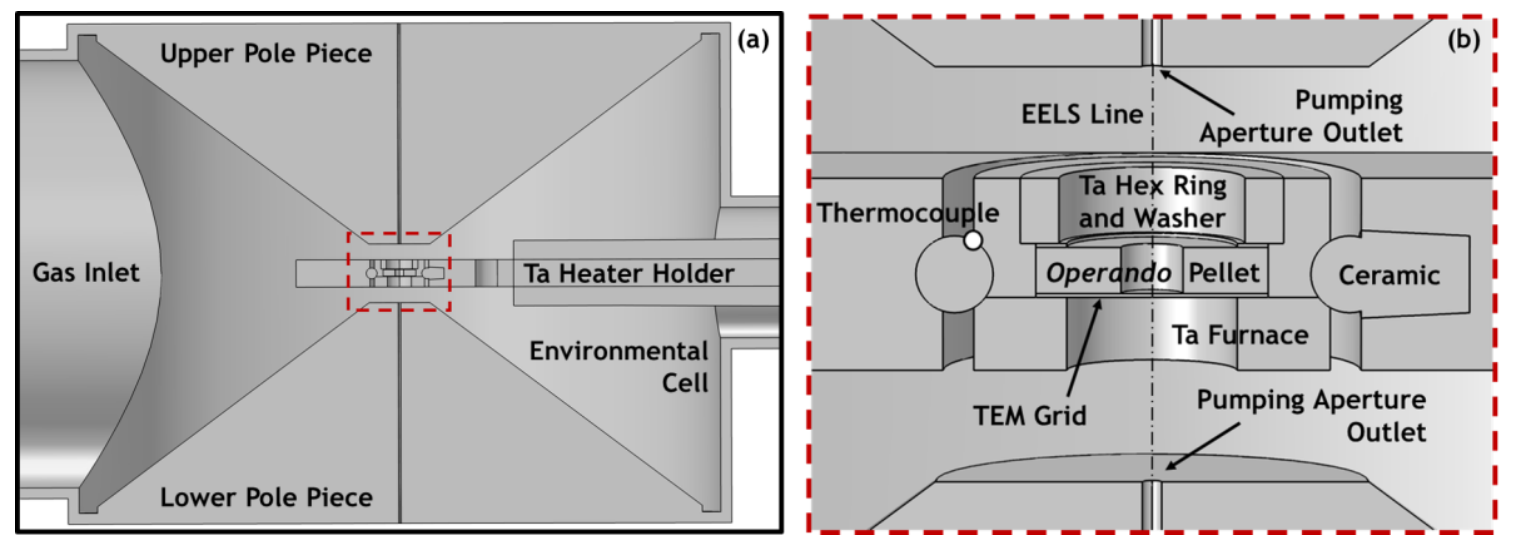

Figure 1. Environmental cell model geometry (a) in full view and (b) in an enhanced view of the operando pellet reactor. The electron beam path is shown with the dashed line labeled "EELS Line".
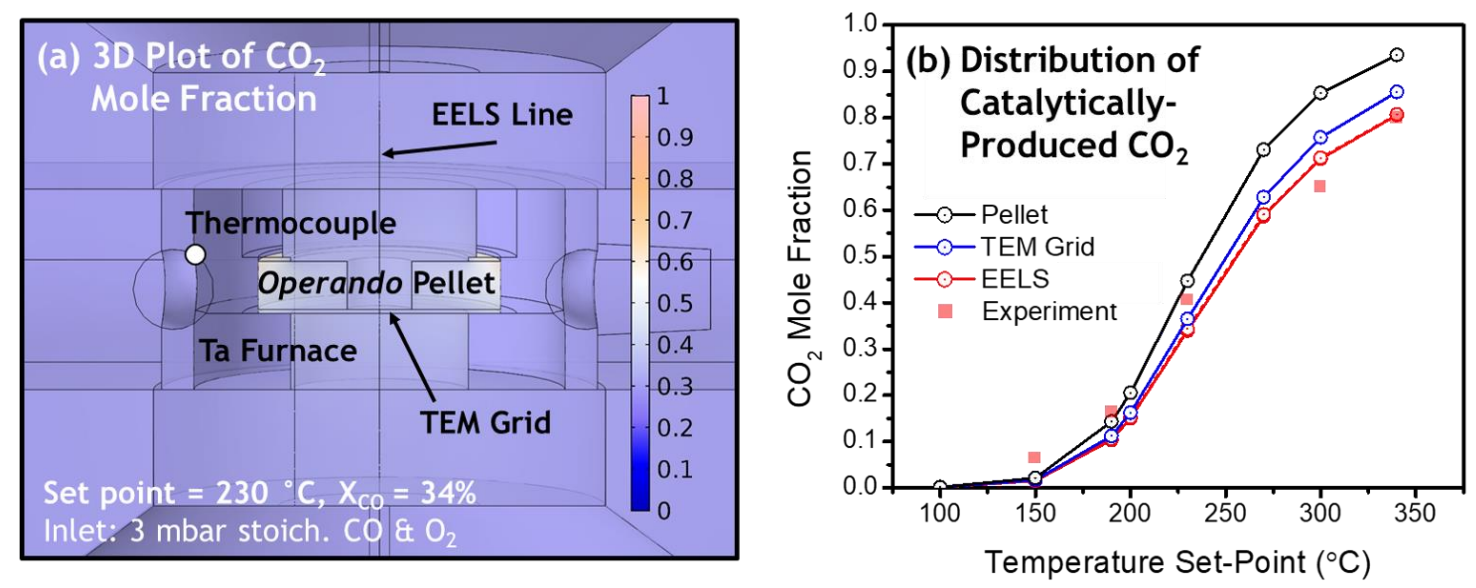

Figure 2. (a) 3D plot of the steady-state mole fraction of catalytically-produced $\mathrm{CO}_{2}$ around the operando pellet reactor for a furnace thermocouple set point of $230{ }^{\circ} \mathrm{C}$, and (b) evaluation of $\mathrm{CO}_{2}$ mole fraction at different domains compared with experimental data across a range of conversions.

\section{References}

[1] Crozier, P. A., and Hansen, T. W. MRS Bulletin, 2015, 40, pp. 38-45.

[2] Chenna, S., and Crozier, P. A. ACS Catalysis, 2012, 2(11), pp. 2395-2402. 
[3] Miller, B. K., Barker, T. M., and Crozier, P. A. Ultramicroscopy, 2015, 156, pp. 18-22.

[4] Mortensen, P. M., et al. Ultramicroscopy, 2015, 152, pp. 1-9.

[5] Miller, B. K., and Crozier, P. A. Microscopy and Microanalysis, 2014, 2(30), pp. 815-824.

[6] We gratefully acknowledge the support of NSF grants CBET-1134464 and CBET-1604971, as well as ASU's John M. Cowley Center for High Resolution Electron Microscopy. 